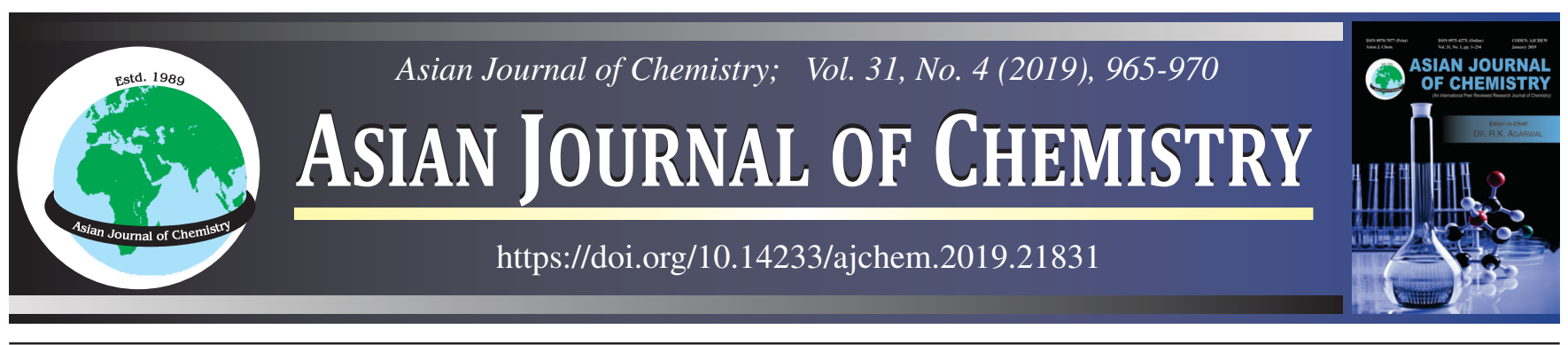

\title{
Synthesis and Preliminary Evaluation of Benzofuran-Oxadiazole Conjugates as Potential Antitubercular Agents
}

\section{Veerabhadrayya S. Negalurmath ${ }^{1}$, Obelannavar Kotresh ${ }^{1,{ }^{*}}$ and Mahantesha Basanagouda ${ }^{2,0}$}

${ }^{1}$ Department of Chemistry, Karnatak Science College, Dharwad-580001, India

${ }^{2}$ P.G. Department of Chemistry, P.C. Jabin Science College, Hubballi-580031, India

*Corresponding author: E-mail: kotresh.obelannavar@gmail.com

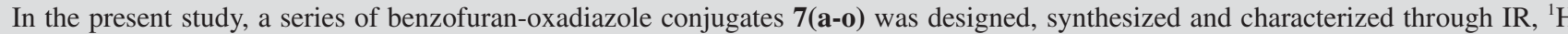
NMR, ${ }^{13} \mathrm{C}$ NMR and mass spectral data. All the compounds were screened for preliminary antitubercular activity against Mycobacterium phlei and Mycobacterium tuberculosis $\mathrm{H}_{37} \mathrm{RV}$. Among all the target compounds, the compound possessing chlorine (7k, MIC $1.56 \mu \mathrm{g} / \mathrm{mL}$ ) and bromine $(7 \mathrm{~m}$, MIC $1.56 \mu \mathrm{g} / \mathrm{mL})$ on $6^{\text {th }}$ position of benzofuran showed highest activity against Mycobacterium phlei. Whereas, bromine on either $5^{\text {th }}$ position (7l, MIC $\left.3.125 \mu \mathrm{g} / \mathrm{mL}\right)$ or $6^{\text {th }}$ position $(7 \mathrm{~m}$ MIC $3.125 \mu \mathrm{g} / \mathrm{mL})$ on benzofuran exhibited highest activity for Mycobacterium tuberculosis $\left(\mathrm{H}_{37} \mathrm{RV}\right)$.

Keywords: Antituberculosis, Benzofuran, Oxadiazole, SAR.

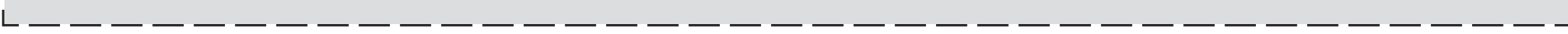

\section{INTRODUCTION}

Tuberculosis (TB) is an old disease, which is caused by Mycobacterium tuberculosis (Mtb). Worldwide, tuberculosis is one of the top 10 causes of death and the leading cause from a single infectious agent (above HIV/AIDS). Millions of people continue to fall sick with tuberculosis each year [1]. During the last decade there has been an increased interest for research on tuberculosis by international and national organizations, pharmaceutical companies. The innovative focus on tuberculosis has partly been prompted by the persistent larger number of tuberculosis case studies in developing countries and partly by the increased occurrence of multidrug and extensively drug resistant tuberculosis (MDR- and XDR-TB) [2,3]. According to WHO 2018 report, in 2017, tuberculosis caused an estimated 1.3 million deaths among HIV-negative people and there were an additional 300000 deaths from tuberculosis among HIV-positive people. Globally, the best estimate is that 10 million people developed tuberculosis disease in 2017: 5.8 million men, 3.2 million women and 1.0 million children [1]. Hence, the need for search the new and efficient antituberculosis agents with a new mechanism of action remains a crucial task $[4,5]$.
Benzofuran and its derivatives are important basis for drug discovery and possessing broad spectrum of biological and pharmaceutical activities [6-11]. The 3-substituted benzofurans have attracted the medicinal chemists due to their exciting biological properties such as antifungal and antitubercular agents [12], antiviral and antitumor [13], cytotoxicity [14], hepatitis $C$ virus inhibitors [15], inhibitors of mycobacterium protein tyrosine phosphatase [16], dual 5- $\mathrm{HT}_{1 \mathrm{~A}}$ receptor and serotonin transporter affinity [17], bone morphogenetic protein2 up-regulators [18], glycogen synthase kinase $3 \beta$ inhibitors [19], calcium activated chloride channel inhibitors [20], inhibition of $A \beta$ neurotoxicity, cholinesterase activity and $\beta$-amyloid aggregation [21], ischemic cell death inhibitors [22], orally bioavailable GPR40 agonist [23]. The potent antitubercular properties exhibited by some compounds with benzofuran [2430] and 3-substituted benzofuran moieties [31-36] are reported in the literature.

Recently, there are many reports pertaining to biological activity of 1,3,4-oxadiazole as antitubercular [37], anticonvulsant [38], antiallergic [39], antiepileptic [40], cytotoxic and antimicrobial [41] and anticancer [42] agents.

The continued attempt of our group on designing oxygen based heterocycles as biologically effective molecules [43-

This is an open access journal, and articles are distributed under the terms of the Creative Commons Attribution-NonCommercial-ShareAlike 4.0 (CC BY-NC-SA 4.0) International License which allows readers to freely read, download, copy, distribute, print, search, or link to the full texts of its articles and to use them for any other lawful non-commercial purpose as long as the original source is duly acknowledged. 
45], here we are reporting the design and synthesis of

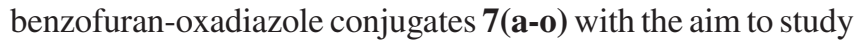
structure activity relationships and thereby provide novel compounds as potential antitubercular agents against Mycobacterium phlei and Mycobacterium tuberculosis $\mathrm{H}_{37} \mathrm{RV}$.

\section{EXPERIMENTAL}

The melting points were determined by open capillary method and are uncorrected. The IR spectra ( $\mathrm{KBr}$ disc) were recorded on a Thermo Fisher Nicolet-6700 FT-IR spectrophotometer. ${ }^{1} \mathrm{H}$ NMR and ${ }^{13} \mathrm{C}$ NMR spectra were recorded on $500 \mathrm{MHz}$ Bruker spectrometer using dimethylsulfoxide $\left(\right.$ DMSO- $d_{6}$ ) as solvent and tetramethylsilane (TMS) as an internal standard. The chemical shifts were expressed in $\delta \mathrm{ppm}$ and coupling constant $(J)$ values were given in Hertz. The mass spectra were recorded using Shimadzu GCMS-QP2010S instrument. The elemental analysis was carried out using Heraus $\mathrm{CHN}$ rapid analyzer. Progress of the reaction was monitored by TLC using aluminium sheets precoated with UV fluorescent silica gel Merck 60 F254 and were visualized by using UV lamp. All the chemicals of analytical grade were purchased from Sigma-Aldrich Chemicals (India) and S.D. Fine Chemicals (India) and were used without further purification unless otherwise stated.

General procedure for synthesis of benzofuran-oxadiazole conjugates 7(a-o): The required benzofuran-3-yl-acetic acid hydrazides $\mathbf{6}(\mathbf{a}-\mathbf{0})$ were prepared according our earlier report [45]. A mixture of carbohydrazide $\mathbf{6}(\mathbf{a}-\mathbf{0})(10 \mathrm{mmol})$ and triethyl orthoformate $(2 \mathrm{~mL})$ in toluene $(60 \mathrm{~mL})$ was heated under reflux for $15 \mathrm{~h}$. The excess solvent was evaporated and the reaction mass was cooled to room temperature. The resultant solid $\mathbf{7 ( a - o )}$ was filtered and recrystallized from ethanol.

2-((5-Methylbenzofuran-3-yl)methyl)-1,3,4-oxadiazole (7a): Colourless solid; m.p. 147-148 ${ }^{\circ} \mathrm{C}$; $(74 \%)$; IR ( $\mathrm{KBr}$, $\left.v_{\max }, \mathrm{cm}^{-1}\right): 1606(\mathrm{C}=\mathrm{N}) ;{ }^{1} \mathrm{H}$ NMR $\left(500 \mathrm{MHz}, \mathrm{DMSO}-d_{6}\right): \delta$ $2.41\left(s, 3 \mathrm{H}, 5-\mathrm{CH}_{3}\right), 3.76\left(s, 2 \mathrm{H}, \mathrm{C} 3-\mathrm{CH}_{2}\right), 7.14-7.16(d d, J=$ $8.5 \mathrm{~Hz}, 1.0 \mathrm{~Hz}, 1 \mathrm{H}, \mathrm{C} 6-\mathrm{H}), 7.41(s, 2 \mathrm{H}, \mathrm{C} 4-\mathrm{H}$ and oxadiazole$\mathrm{H}), 7.45(d, J=8.5 \mathrm{~Hz}, \mathrm{C} 7-\mathrm{H}), 7.80(s, 1 \mathrm{H}, \mathrm{C} 2-\mathrm{H}) ;{ }^{13} \mathrm{C}$ NMR (125 MHz, DMSO- $d_{6}$ ): $\delta 20.12,21.68,111.82,113.84,120.18$, 124.42, 125.56, 134.74, 143.28, 155.42, 170.90; GCMS $m / z$ : 214 [M+]; Anal. calcd. for $\mathrm{C}_{12} \mathrm{H}_{10} \mathrm{~N}_{2} \mathrm{O}_{2} ; \mathrm{C}, 67.28 ; \mathrm{H}, 4.71 ;$; , 13.08; Found: C, 67.26; H, 4.71; N, 13.07.

2-((6-Methylbenzofuran-3-yl)methyl)-1,3,4-oxadiazole (7b): Colourless solid; m.p. $173-174{ }^{\circ} \mathrm{C}$; yield (76\%); IR (KBr, $\left.v_{\max }, \mathrm{cm}^{-1}\right): 1606(\mathrm{C}=\mathrm{N}) ;{ }^{1} \mathrm{H}$ NMR $\left(500 \mathrm{MHz}, \mathrm{DMSO}-d_{6}\right): \delta$ $2.40\left(s, 3 \mathrm{H}, 6-\mathrm{CH}_{3}\right), 3.73\left(s, 2 \mathrm{H}, \mathrm{C} 3-\mathrm{CH}_{2}\right), 7.06-7.08(d, J=$ $8.0 \mathrm{~Hz}, 1 \mathrm{H}, \mathrm{C} 5-\mathrm{H}), 7.35$ ( $s, 2 \mathrm{H}, \mathrm{C} 7-\mathrm{H}$ and oxadiazole-H), 7.43$7.45(d, J=8.0 \mathrm{~Hz}, 1 \mathrm{H}, \mathrm{C} 4-\mathrm{H}), 7.79(s, 1 \mathrm{H}, \mathrm{C} 2-\mathrm{H}) ;{ }^{13} \mathrm{C}$ NMR (125 MHz, DMSO- $\left.d_{6}\right): \delta 19.29,21.63,111.78,113.78,120.03$, $124.39,125.54,134.66,143.25,155.39,170.87$; GCMS $m / z$ : 213 [M+1]; Anal. calcd. for $\mathrm{C}_{12} \mathrm{H}_{10} \mathrm{~N}_{2} \mathrm{O}_{2} ; \mathrm{C}, 67.28 ; \mathrm{H}, 4.71$; N, 13.08; Found: C, 67.27; H, 4.71; N, 13.07.

2-((4,6-Dimethylbenzofuran-3-yl)methyl)-1,3,4oxadiazole (7c): Colourless; m.p. $167-168{ }^{\circ} \mathrm{C}$, yield (72\%); IR $\left(\mathrm{KBr}, v_{\max }, \mathrm{cm}^{-1}\right) 1619(\mathrm{C}=\mathrm{N})$; ${ }^{1} \mathrm{H}$ NMR $(500 \mathrm{MHz}$, DMSO$\left.d_{6}\right): \delta 2.35\left(s, 3 \mathrm{H}, \mathrm{CH}_{3}\right), 2.46\left(s, 3 \mathrm{H}, \mathrm{CH}_{3}\right), 3.75(s, 2 \mathrm{H}, \mathrm{C} 3-$ $\left.\mathrm{CH}_{2}\right), 6.80(s, 1 \mathrm{H}, \mathrm{C} 5-\mathrm{H}), 7.19(s, 1 \mathrm{H}, \mathrm{C} 7-\mathrm{H}), 7.28(\mathrm{~s}, 1 \mathrm{H}$,
oxadiazole-H), $7.82(s, 1 \mathrm{H}, \mathrm{C} 2-\mathrm{H}) ;{ }^{13} \mathrm{CNMR}(125 \mathrm{MHz}$, DMSO$\left.d_{6}\right): 19.08,21.48,21.94,110.94,113.65,120.14,124.44$, 126.02, 134.98, 143.56, 155.99, 164.72, 171.10; GCMS m/z: 228 [M+]; Anal. calcd. for $\mathrm{C}_{13} \mathrm{H}_{12} \mathrm{~N}_{2} \mathrm{O}_{2} ; \mathrm{C}, 68.41 ; \mathrm{H}, 5.30 ; \mathrm{N}$, 12.27; Found: C, 68.40; H, 5.30; N, 12.26.

2-((6,7-Dimethylbenzofuran-3-yl)methyl)-1,3,4oxadiazole (7d): Colourless solid; m.p. $154-155^{\circ} \mathrm{C}$; yield (70 $\%)$; IR ( $\left.\mathrm{KBr}, v_{\max }, \mathrm{cm}^{-1}\right): 1620(\mathrm{C}=\mathrm{N})$; ${ }^{1} \mathrm{H} \mathrm{NMR}(500 \mathrm{MHz}$, DMSO- $\left.d_{6}\right): \delta 2.34\left(s, 3 \mathrm{H}, \mathrm{CH}_{3}\right), 2.45\left(s, 3 \mathrm{H}, \mathrm{CH}_{3}\right), 3.78(s$, $\left.2 \mathrm{H}, \mathrm{C} 3-\mathrm{CH}_{2}\right), 7.08-7.10(d, J=8.0 \mathrm{~Hz}, 1 \mathrm{H}, \mathrm{C} 5-\mathrm{H}), 7.24-7.26$ $(d, J=8.0 \mathrm{~Hz}, 1 \mathrm{H}, \mathrm{C} 4-\mathrm{H}), 7.45(s, 1 \mathrm{H}$, oxadiazole- $\mathrm{H}), 7.78$ $(s, 1 \mathrm{H}, \mathrm{C} 2-\mathrm{H}) ;{ }^{13} \mathrm{C}$ NMR $\left(125 \mathrm{MHz}\right.$, DMSO- $\left.d_{6}\right): \delta 18.65,19.94$, 23.24, 110.63, 113.21, 123.10, 125.27, 125.64, 130.60, 142.11, 155.67, 166.92, 171.06; GCMS m/z: 228 [M+]; Anal. calcd. for $\mathrm{C}_{13} \mathrm{H}_{12} \mathrm{~N}_{2} \mathrm{O}_{2} ; \mathrm{C}, 68.41 ; \mathrm{H}, 5.30 ; \mathrm{N}, 12.27$; Found: C, 68.39; $\mathrm{H}, 5.30 ; \mathrm{N}, 12.25$.

2-((5-iso-Propylbenzofuran-3-yl)methyl)-1,3,4oxadiazole (7e): Colourless solid; m.p. $142-143^{\circ} \mathrm{C}$; yield (72 $\%)$; IR ( $\left.\mathrm{KBr}, v_{\max }, \mathrm{cm}^{-1}\right): 1630(\mathrm{C}=\mathrm{N})$; ${ }^{1} \mathrm{H}$ NMR $(500 \mathrm{MHz}$, DMSO- $\left.d_{6}\right): \delta 1.27\left(d, 6 \mathrm{H}\right.$, isopropyl- $\left.\mathrm{CH}_{3}, J=6 \mathrm{~Hz}\right), 2.95-$ $3.07\left(m, 1 \mathrm{H}\right.$, isopropyl-CH), $3.76\left(s, 2 \mathrm{H}, \mathrm{C} 3-\mathrm{CH}_{2}\right), 7.11(d d, J$ $=8.5 \mathrm{~Hz}, 1.5 \mathrm{~Hz}, 1 \mathrm{H}, \mathrm{C} 6-\mathrm{H}), 7.40-7.45(\mathrm{~m}, 2 \mathrm{H}, \mathrm{C} 4-\mathrm{H}$ and $\mathrm{C} 7-$ $\mathrm{H}), 7.60\left(s, 1 \mathrm{H}\right.$, oxadiazole-H), $7.75(s, 1 \mathrm{H}, \mathrm{C} 2-\mathrm{H}) ;{ }^{13} \mathrm{C}$ NMR $\left(125 \mathrm{MHz}, \mathrm{DMSO}-d_{6}\right): \delta 23.10,29.52,34.06,111.16,114.58$, $122.26,123.45,129.34,135.18,145.12,156.54,167.63$, 171.20; GCMS m/z: 242 [M+]; Anal. calcd. for $\mathrm{C}_{14} \mathrm{H}_{14} \mathrm{~N}_{2} \mathrm{O}_{2}$; C, 69.38; H, 5.82; N, 11.54; Found: C, 69.36; H, 5.82; N, 11.53 .

2-((5-tert-Butylbenzofuran-3-yl)methyl)-1,3,4oxadiazole (7f): Colourless solid; m.p. 163-164 ${ }^{\circ} \mathrm{C}$; yield (75 $\%)$; IR $\left(\mathrm{KBr}, v_{\max }, \mathrm{cm}^{-1}\right): 1620(\mathrm{C}=\mathrm{N}) ;{ }^{1} \mathrm{H}$ NMR $(500 \mathrm{MHz}$, DMSO- $\left.d_{6}\right): \delta 1.38\left(s, 9 \mathrm{H}, \mathrm{CH}_{3}\right), 3.84\left(s, 2 \mathrm{H}, \mathrm{C}_{3}-\mathrm{CH}_{2}\right), 7.10$ $(d d, J=8.5 \mathrm{~Hz}, 1.5 \mathrm{~Hz}, 1 \mathrm{H}, \mathrm{C} 6-\mathrm{H}), 7.44-7.50(m, 2 \mathrm{H}, \mathrm{C} 4-\mathrm{H}$, C7-H and oxadiazole-H), $7.81(s, 1 \mathrm{H}, \mathrm{C} 2-\mathrm{H}) ;{ }^{13} \mathrm{C}$ NMR $(125$ MHz, DMSO- $d_{6}$ ): 29.40, 31.56, 44.56, 114.15, 117.32, 121.45, 126.94, 128.68, 131.68, 144.36, 157.14, 167.38. 171.33; GCMS m/z: $256[\mathrm{M}+]$; Anal. calcd. for $\mathrm{C}_{15} \mathrm{H}_{16} \mathrm{~N}_{2} \mathrm{O}_{2} ; \mathrm{C}, 70.29$; H, 6.29; N, 10.93; Found: C, 70.27; H, 6.29; N, 10.92 .

3-((1,3,4-Oxadiazol-2-yl)methyl)benzofuran-6-ol (7g): Colourless solid; m.p. $150-151{ }^{\circ} \mathrm{C}$; yield $(73 \%)$; IR $(\mathrm{KBr}$, $\left.v_{\max }, \mathrm{cm}^{-1}\right): 1632(\mathrm{C}=\mathrm{N}), 3256(\mathrm{Broad}, \mathrm{OH}) ;{ }^{1} \mathrm{H}$ NMR $(500$ $\left.\mathrm{MHz}, \mathrm{DMSO}-d_{6}\right): \delta 3.86\left(s, 2 \mathrm{H}, \mathrm{C} 3-\mathrm{CH}_{2}\right), 6.76(d d, J=8.4$ $\mathrm{Hz}, 2.0 \mathrm{~Hz}, 1 \mathrm{H}, \mathrm{C} 5-\mathrm{H}), 6.86-6.88(d, J=2.0 \mathrm{~Hz}, 1 \mathrm{H}, \mathrm{C} 7-\mathrm{H})$, $7.36(d, J=8.4 \mathrm{~Hz}, 1 \mathrm{H}, \mathrm{C} 4-\mathrm{H}), 7.43(\mathrm{~m}, 2 \mathrm{H}$, oxadiazole- $\mathrm{H}$ and $\mathrm{C} 2-\mathrm{H}) ;{ }^{13} \mathrm{C}$ NMR $\left(125 \mathrm{MHz}\right.$, DMSO- $\left.d_{6}\right): \delta 29.50,99.74$, 114.30, 116.66, 120.24, 123.92, 144.84, 158.30, 158.64, 167.80, 172.18; GCMS m/z: 216 [M+]; Anal. calcd. for $\mathrm{C}_{11} \mathrm{H}_{8} \mathrm{~N}_{2} \mathrm{O}_{3} ; \mathrm{C}, 61.11 ; \mathrm{H}, 3.73 ; \mathrm{N}, 12.96$; Found: $\mathrm{C}, 61.09 ; \mathrm{H}$, $3.73 ; \mathrm{N}, 12.95$.

2-((5-Methoxybenzofuran-3-yl)methyl)-1,3,4-oxadiazole (7h): Beige solid; m.p. $146-147^{\circ} \mathrm{C}$; yield (82\%); IR (KBr, $\left.v_{\max }, \mathrm{cm}^{-1}\right): 1618(\mathrm{C}=\mathrm{N}) ;{ }^{1} \mathrm{H}$ NMR (500 MHz, DMSO- $\left.d_{6}\right): \delta$ $3.58\left(s, 3 \mathrm{H}, 5-\mathrm{OCH}_{3}\right), 3.89\left(s, 2 \mathrm{H}, \mathrm{C} 3-\mathrm{CH}_{2}\right), 6.85-6.91(d d, J$ $=8.0 \mathrm{~Hz}, 2.0 \mathrm{~Hz}, 1 \mathrm{H}, \mathrm{C} 6-\mathrm{H}), 7.25(d, J=2.0 \mathrm{~Hz}, 1 \mathrm{H}, \mathrm{C} 4-\mathrm{H})$, $7.41(d, J=8.0 \mathrm{~Hz}, 1 \mathrm{H}, \mathrm{C} 7-\mathrm{H}), 7.56(s, 1 \mathrm{H}$, oxadiazole- $\mathrm{H})$, $7.83(s, 1 \mathrm{H}, \mathrm{C} 2-\mathrm{H}) ;{ }^{13} \mathrm{C}$ NMR (125 MHz, DMSO- $\left.d_{6}\right): \delta 29.84$, 57.12, 106.18, 115.10, 115.63, 115.79, 128.12, 144.58, 151.35, 156.94, 168.87, 172.33; GCMS m/z: 230 [M+]; Anal. calcd. 
for $\mathrm{C}_{12} \mathrm{H}_{10} \mathrm{~N}_{2} \mathrm{O}_{3} ; \mathrm{C}, 62.60 ; \mathrm{H}, 4.38 ; \mathrm{N}, 12.17$; Found: $\mathrm{C}, 62.54$; $\mathrm{H}, 4.38 ; \mathrm{N}, 12.16$.

2-((6-Methoxybenzofuran-3-yl)methyl)-1,3,4-oxadiazole (7i): Grey solid; m.p. $171-172{ }^{\circ} \mathrm{C}$; yield $(80 \%)$; IR (KBr, $\left.v_{\max }, \mathrm{cm}^{-1}\right): 1627(\mathrm{C}=\mathrm{N}) ;{ }^{1} \mathrm{H}$ NMR $\left(500 \mathrm{MHz}, \mathrm{DMSO}-d_{6}\right): \delta$ $3.59\left(s, 3 \mathrm{H}, 6-\mathrm{OCH}_{3}\right), 3.89\left(s, 2 \mathrm{H}, \mathrm{C} 3-\mathrm{CH}_{2}\right), 6.86(d d, J=9.0$ $\mathrm{Hz}, 2.0 \mathrm{~Hz}, 1 \mathrm{H}, \mathrm{C} 5-\mathrm{H}), 7.14(d, J=2.0 \mathrm{~Hz}, 1 \mathrm{H}, \mathrm{C} 7-\mathrm{H}), 7.44$ $(d, J=9.0 \mathrm{~Hz}, 1 \mathrm{H}, \mathrm{C} 4-\mathrm{H}), 7.61(s, 1 \mathrm{H}$, oxadiazole-H), 7.78 $(s, 1 \mathrm{H}, \mathrm{C} 2-\mathrm{H}) ;{ }^{13} \mathrm{C}$ NMR $\left(125 \mathrm{MHz}, \mathrm{DMSO}-d_{6}\right): \delta 21.70,56.14$, 104.55, 105.34, 112.98, 114.14, 125.38, 144.17, 144.54, 169.05, 172.06; GCMS m/z: 230 [M+]; Anal. calcd. for $\mathrm{C}_{12} \mathrm{H}_{10} \mathrm{~N}_{2} \mathrm{O}_{3} ; \mathrm{C}, 62.60 ; \mathrm{H}, 4.38 ; \mathrm{N}, 12.17$; Found: $\mathrm{C}, 62.58 ; \mathrm{H}$, $4.38 ; \mathrm{N}, 12.16$.

2-((5-Chlorobenzofuran-3-yl)methyl)-1,3,4-oxadiazole (7j): Colourless solid; m.p. $149-150{ }^{\circ} \mathrm{C}$; yield $(69 \%)$; IR (KBr, $\left.v_{\max }, \mathrm{cm}^{-1}\right): 1639(\mathrm{C}=\mathrm{N}) ;{ }^{1} \mathrm{H}$ NMR $\left(500 \mathrm{MHz}, \mathrm{DMSO}-d_{6}\right): \delta$ $3.92\left(s, 2 \mathrm{H}, \mathrm{C} 3-\mathrm{CH}_{2}\right), 7.36(d, J=9.0 \mathrm{~Hz}, 1 \mathrm{H}, \mathrm{C} 6-\mathrm{H}), 7.59(d$, $J=9.0 \mathrm{~Hz}, 1 \mathrm{H}, \mathrm{C} 7-\mathrm{H}), 7.64(s, 2 \mathrm{H}, \mathrm{C} 4-\mathrm{H}$ and oxadiazole-H), $7.90(s, 1 \mathrm{H}, \mathrm{C} 2-\mathrm{H}) ;{ }^{13} \mathrm{C}$ NMR $\left(125 \mathrm{MHz}, \mathrm{DMSO}-d_{6}\right): 29.71$, 114.18, 115.92, 121.44, 125.56, 128.01, 131.35, 145.98, 155.33, 168.12, 171.32; GCMS m/z: 234, 236 [M+, M+2]; Anal. calcd. for $\mathrm{C}_{11} \mathrm{H}_{7} \mathrm{~N}_{2} \mathrm{O}_{2} \mathrm{Cl} ; \mathrm{C}, 56.31 ; \mathrm{H}, 3.01 ; \mathrm{N}, 11.94$; Found: C, 56.30; H, 3.01; N, 11.93.

2-((6-Chlorobenzofuran-3-yl)methyl)-1,3,4-oxadiazole (7k): Colourless solid; m.p. $158-159^{\circ} \mathrm{C}$; yield $(67 \%)$; IR (KBr, $\left.v_{\max }, \mathrm{cm}^{-1}\right): 1631(\mathrm{C}=\mathrm{N}) ;{ }^{1} \mathrm{H}$ NMR $\left(500 \mathrm{MHz}, \mathrm{DMSO}-d_{6}\right): \delta$ $3.90\left(s, 2 \mathrm{H}, \mathrm{C} 3-\mathrm{CH}_{2}\right), 7.09-7.11(d, J=8.0 \mathrm{~Hz}, 1 \mathrm{H}, \mathrm{C} 5-\mathrm{H})$, $7.42(s, 1 \mathrm{H}, \mathrm{C} 7-\mathrm{H}), 7.44-7.46(d, J=8.0 \mathrm{~Hz}, 1 \mathrm{H}, \mathrm{C} 4-\mathrm{H}), 7.51$ $(s, 1 \mathrm{H}$, oxadiazole- $\mathrm{H}), 7.76(s, 1 \mathrm{H}, \mathrm{C} 2-\mathrm{H}) ;{ }^{13} \mathrm{C}$ NMR $(125 \mathrm{MHz}$, DMSO- $d_{6}$ ): $\delta 29.98,115.33,116.54,121.98,125.74,128.45$, 132.44, 147.58, 156.10, 166.41, 172.43; GCMS m/z: 234, 236 $[\mathrm{M}+, \mathrm{M}+2]$; Anal. calcd. for $\mathrm{C}_{11} \mathrm{H}_{7} \mathrm{~N}_{2} \mathrm{O}_{2} \mathrm{Cl} ; \mathrm{C}, 56.31 ; \mathrm{H}, 3.01$; N, 11.94; Found: C, 56.30; H, 3.01; N, 11.93 .

2-((5-Bromobenzofuran-3-yl)methyl)-1,3,4-oxadiazole (7l): Colourless solid; m.p. $168-169^{\circ} \mathrm{C}$; yield $(66 \%)$; IR (KBr, $\left.v_{\max }, \mathrm{cm}^{-1}\right): 1635(\mathrm{C}=\mathrm{N}) ;{ }^{1} \mathrm{H}$ NMR $\left(500 \mathrm{MHz}, \mathrm{DMSO}-d_{6}\right): \delta$ $3.91\left(s, 2 \mathrm{H}, \mathrm{C} 3-\mathrm{CH}_{2}\right), 7.41-7.44(d d, J=9.0 \mathrm{~Hz}, 2.0 \mathrm{~Hz}, 1 \mathrm{H}$, C6-H), $7.53(d, J=9.0 \mathrm{~Hz}, 1 \mathrm{H}, \mathrm{C} 7-\mathrm{H}), 7.86(d, J=2.0 \mathrm{~Hz}$, $1 \mathrm{H}, \mathrm{C} 4-\mathrm{H}), 7.88-7.90(m, 2 \mathrm{H}$, oxadiazole- $\mathrm{H}$ and $\mathrm{C} 2-\mathrm{H}) ;{ }^{13} \mathrm{C}$ NMR (125 MHz, DMSO-d $\left.d_{6}\right): \delta 29.52,114.04,115.98,116.07$, $124.53,128.33,132.84,146.78,155.62,164.47,172.14$; GCMS m/z: 277, $279[\mathrm{M}+, \mathrm{M}+2]$; Anal. calcd. for $\mathrm{C}_{11} \mathrm{H}_{7} \mathrm{~N}_{2} \mathrm{O}_{2} \mathrm{Br} ; \mathrm{C}$, 47.34; H, 2.53; N, 10.04; Found: C, 47.33; $\mathrm{H}, 2.53 ; \mathrm{N}, 10.04$.

2-((6-Bromobenzofuran-3-yl)methyl)-1,3,4-oxadiazole (7m): Colourless solid; m.p. $134-135{ }^{\circ} \mathrm{C}$; yield $(60 \%)$; IR $\left(\mathrm{KBr}, v_{\max }, \mathrm{cm}^{-1}\right): 1629(\mathrm{C}=\mathrm{N})$; ${ }^{1} \mathrm{H}$ NMR $(500 \mathrm{MHz}$, DMSO$\left.d_{6}\right): \delta 3.91\left(s, 2 \mathrm{H}, \mathrm{C} 3-\mathrm{CH}_{2}\right), 7.08-7.10(d, J=8.0 \mathrm{~Hz}, 1 \mathrm{H}, \mathrm{C} 5-$ $\mathrm{H}), 7.39(s, 1 \mathrm{H}, \mathrm{C} 7-\mathrm{H}), 7.42-7.44(d, J=8.0 \mathrm{~Hz}, 1 \mathrm{H}, \mathrm{C} 4-\mathrm{H})$, $7.60\left(s, 1 \mathrm{H}\right.$, oxadiazole-H), $7.75(s, 1 \mathrm{H}, \mathrm{C} 2-\mathrm{H}) ;{ }^{13} \mathrm{C}$ NMR $(125$ MHz, DMSO- $\left.d_{6}\right)$ : 29.98, 115.11, 116.47, 121.52, 127.45, 129.82, 135.84, 148.44, 156.87, 169.33, 172.70; GCMS m/z: 277, 279 [M+, M+2]; Anal. calcd. for $\mathrm{C}_{11} \mathrm{H}_{7} \mathrm{~N}_{2} \mathrm{O}_{2} \mathrm{Br}$; C, 47.34; H, 2.53; N, 10.04; Found: C, 47.32; H, 2.53; N, 10.04.

2-(Naphtho[2,1-b]furan-1-ylmethyl)-1,3,4-oxadiazole (7n): Beige solid; m.p. $201-202{ }^{\circ} \mathrm{C}$; yield (72 \%); IR (KBr, $\left.v_{\max }, \mathrm{cm}^{-1}\right): 1618(\mathrm{C}=\mathrm{N}) ;{ }^{1} \mathrm{H}$ NMR $\left(500 \mathrm{MHz}\right.$, DMSO- $\left.d_{6}\right): \delta$ $3.81\left(s, 2 \mathrm{H}, \mathrm{C} 3-\mathrm{CH}_{2}\right), 7.46-7.49(t, J=7.5 \mathrm{~Hz}, 1 \mathrm{H}, \mathrm{Ar}-\mathrm{H})$,
7.52-7.55 $(t, J=7.5 \mathrm{~Hz}, 1 \mathrm{H}, \mathrm{Ar}-\mathrm{H}), 7.78-7.80(d, J=9.0 \mathrm{~Hz}$, $1 \mathrm{H}, \mathrm{Ar}-\mathrm{H}), 7.86-7.88(d, J=9.0 \mathrm{~Hz}, 1 \mathrm{H}, \mathrm{Ar}-\mathrm{H}), 8.05-8.10(m$, $2 \mathrm{H}$, oxadiazole- $\mathrm{H}$ and $\mathrm{Ar}-\mathrm{H}), 8.10(s, 1 \mathrm{H}, \mathrm{C} 2-\mathrm{H}), 8.14-8.15$ $(d, J=8.5 \mathrm{~Hz}, 1 \mathrm{H}, \mathrm{Ar}-\mathrm{H}) ;{ }^{13} \mathrm{C}$ NMR $\left(125 \mathrm{MHz}, \mathrm{DMSO}-d_{6}\right): \delta$ 22.56, 114.12, 114.64, 121.28, 123.87, 126.44, 126.82, 127.60, 127.98, 129.87, 131.74, 145.33, 154.82, 164.74, 172.10; GCMS m/z: $250[\mathrm{M}+]$; Anal. calcd. for $\mathrm{C}_{15} \mathrm{H}_{10} \mathrm{~N}_{2} \mathrm{O}_{2} ; \mathrm{C}, 71.99$; H, 4.03; N, 11.19; Found: C, 71.97; H, 4.03; N, 11.18.

2-(Naphtho[1,2-b]furan-3-ylmethyl)-1,3,4-oxadiazole (7o): Brown solid; m.p. $161-162{ }^{\circ} \mathrm{C}$; yield (70 \%); IR (KBr, $\left.v_{\max }, \mathrm{cm}^{-1}\right): 1652(\mathrm{C}=\mathrm{N}) ;{ }^{1} \mathrm{H}$ NMR $\left(500 \mathrm{MHz}, \mathrm{DMSO}-d_{6}\right): \delta$ $3.86\left(s, 2 \mathrm{H}, \mathrm{C} 3-\mathrm{CH}_{2}\right), 7.46-7.49(m, 1 \mathrm{H}, \mathrm{Ar}-\mathrm{H}), 7.61-7.63(m$, $1 \mathrm{H}, \mathrm{Ar}-\mathrm{H}), 7.65-7.67(d, J=8.4 \mathrm{~Hz}, 1 \mathrm{H}, \mathrm{Ar}-\mathrm{H}), 7.70-7.72(d$, $J=8.8 \mathrm{~Hz}, 1 \mathrm{H}, \mathrm{Ar}-\mathrm{H}), 8.02(\mathrm{~m}, 3 \mathrm{H}$, oxadiazole and Ar-H), $8.18(d, J=8.0 \mathrm{~Hz}, 1 \mathrm{H}, \mathrm{Ar}-\mathrm{H}) ;{ }^{13} \mathrm{C}$ NMR $(125 \mathrm{MHz}$, DMSO$\left.d_{6}\right): \delta 29.68,116.68,119.82,119.99,121.82,124.56,124.18$, 126.63, 128.10, 128.64, 133.18, 145.68, 149.98, 169.15, 172.44; GCMS m/z: 250 [M+]; Anal. calcd. for $\mathrm{C}_{15} \mathrm{H}_{10} \mathrm{~N}_{2} \mathrm{O}_{2}$; C, 71.99; H, 4.03; N, 11.19; Found: C, 71.98; H, 4.03; N, 11.19 .

Antitubercular activity: The antitubercular activity of titled compounds 7(a-o) were performed against M. phlei and M. tuberculosis $\mathrm{H}_{37} \mathrm{RV}$ using well known procedure microplate alamar blue assay (MABA) [46]. This methodology is nontoxic, uses stable reagents and shows good correlation with proportional and BACTEC radiometric method. In brief, 200 $\mu \mathrm{L}$ of sterile deionized water was added to all outer perimeter wells of sterile 96-well plate to minimize evaporation of medium in the test wells during incubation. The 96-well plate received $100 \mu \mathrm{L}$ of the Middlebrook 7H9 broth and serial dilution of compounds was made directly on plate. The final drug concentrations tested were $100-0.1 \mu \mathrm{g} / \mathrm{mL}$. Plates were covered and sealed with parafilm and incubated at $37{ }^{\circ} \mathrm{C}$ for 5 days. After this time, $25 \mu \mathrm{L}$ of freshly prepared 1:1 mixture of Alamar Blue reagent and $10 \%$ Tween 80 were added to the plate and incubated for $24 \mathrm{~h}$. A blue colour in the well was interpreted as no bacterial growth and pink colour was scored as growth. The MIC was defined as the lowest drug concentration that prevented the colour change from blue to pink.

\section{RESULTS AND DISCUSSION}

The concise synthetic route used to synthesize the intermediates and titled compounds 7(a-o) are outlined in Schemes I and II. Benzofuran-3-yl-acetic acids 5(a-o) were converted into corresponding ethyl esters by refluxing with absolute ethanol in presence of conc. sulphuric acid. The resulting mixture, was converted into the acid hydrazides $\mathbf{6}(\mathbf{a - o})$ by the reaction with hydrazine hydrate at reflux temperature [45]. Further, these acid hydrazides $\mathbf{6}(\mathbf{a - 0})$ were reacted with triethyl orthoformate in toluene at reflux temperature for $15 \mathrm{~h}$ afforded title compounds 7(a-0). All the synthesized compounds 7(a-o) were characterized by elemental analysis, IR, ${ }^{1} \mathrm{H} \mathrm{NMR},{ }^{13} \mathrm{C}$ NMR and mass spectral data.

The IR spectrum of an intermediate (6-methyl-benzofuran-3-yl)-acetic acid hydrazide (6b), showed a strong peak at $1643 \mathrm{~cm}^{-1}$ for carbonyl group whereas $3303 \mathrm{~cm}^{-1}$ for $\mathrm{NHNH}_{2}$ stretching. The ${ }^{1} \mathrm{H}$ NMR spectrum exhibited $\delta 2.41(s, 3 \mathrm{H}, 6-$ $\left.\mathrm{CH}_{3}\right), 3.63\left(d, J=1.0 \mathrm{~Hz}, 2 \mathrm{H}, \mathrm{C} 3-\mathrm{CH}_{2}\right), 4.22$ (s, br, 2H, $\mathrm{NH}_{2}$, 


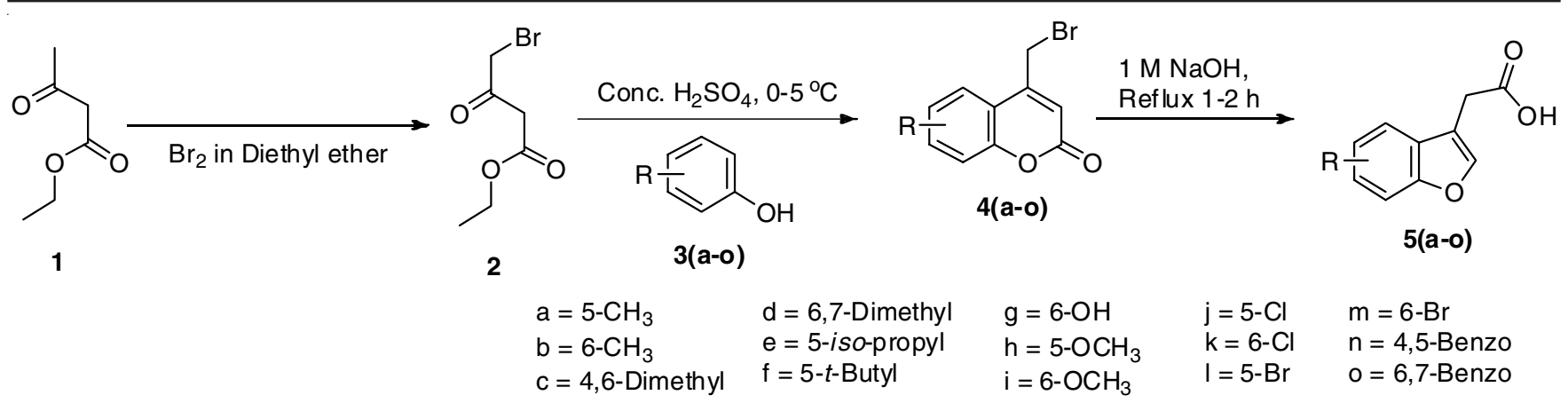

Scheme-I: Synthesis of benzofuran acetic acids 5(a-o)

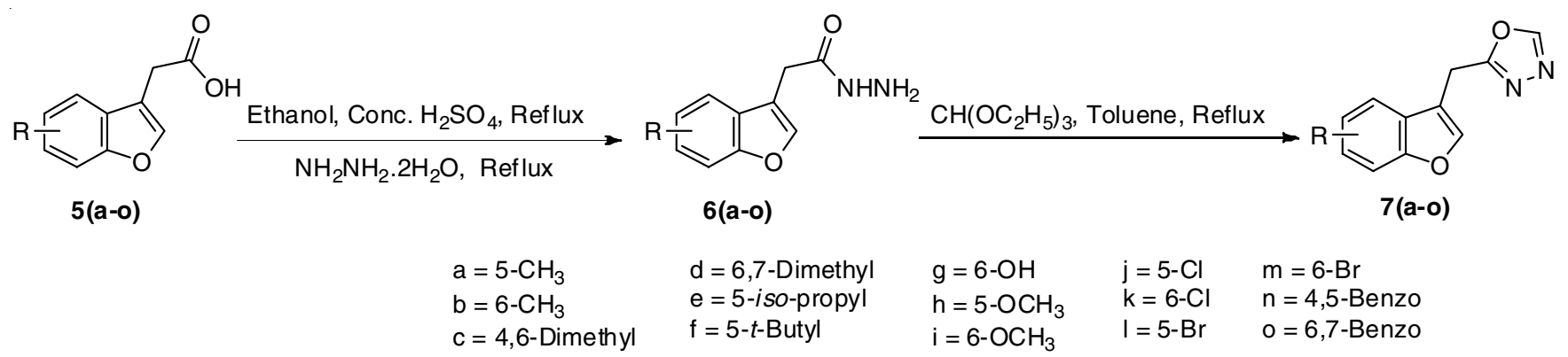

Scheme-II: Synthesis of benzofuran-oxadiazole conjugates 7(a-o)

$\mathrm{D}_{2} \mathrm{O}$ exchangeable), $7.06(d, J=8.0 \mathrm{~Hz}, 1 \mathrm{H}, \mathrm{C} 5-\mathrm{H}), 7.33(s$, $1 \mathrm{H}, \mathrm{C} 7-\mathrm{H}), 7.43(d, J=8.0 \mathrm{~Hz}, 1 \mathrm{H}, \mathrm{C} 4-\mathrm{H}), 7.73(s, 1 \mathrm{H}, \mathrm{C} 2-$ $\mathrm{H}), 9.26$ ( $s$, br, $1 \mathrm{H}, \mathrm{NH}_{1} \mathrm{D}_{2} \mathrm{O}$ exchangeable); which was further confirmed by ${ }^{13} \mathrm{C}$ NMR spectrum agrees with the number of carbons and by its mass spectrum that showed the molecular ion peak $\mathrm{m} / \mathrm{z} 204(\mathrm{M}+)$, confirms the molecular weight of the compound [45].

The IR spectrum of representative compound in the series 2-((6-methylbenzofuran-3-yl)methyl)-1,3,4-oxadiazole (7b), showed absence of carbonyl group and $\mathrm{NHNH}_{2}$ stretching frequencies. Further, new band appeared at $1606(\mathrm{C}=\mathrm{N})$ indicate the cyclization. The ${ }^{1} \mathrm{H}$ NMR spectrum exhibited $\delta 2.40$ $\left(s, 3 \mathrm{H}, 6-\mathrm{CH}_{3}\right), 3.73\left(s, 2 \mathrm{H}, \mathrm{C} 3-\mathrm{CH}_{2}\right), 7.06-7.08(d, J=8.0 \mathrm{~Hz}$, 1H, C5-H), 7.35 ( $s, 2 \mathrm{H}, \mathrm{C} 7-\mathrm{H}$ and oxadiazole-H), 7.43-7.45 $(d, J=8.0 \mathrm{~Hz}, 1 \mathrm{H}, \mathrm{C} 4-\mathrm{H}), 7.79(s, 1 \mathrm{H}, \mathrm{C} 2-\mathrm{H})$; Here, the disappearance of $\mathrm{NH}_{2}$ protons at $4.22\left(s, \mathrm{br}, 2 \mathrm{H}, \mathrm{NH}_{2}, \mathrm{D}_{2} \mathrm{O}\right.$ exchangeable) in the precursor compound indicates the cyclization to the compound $\mathbf{7 b}$. Futher, all the carbons are resonated in the expected regions. The formation of the compound $\mathbf{7 b}$ is confirmed by its mass spectrum which showed molecular ion peak at 213 .

Antitubercular evaluation: The in vitro antitubercular activity against Mycobacterium phlei and Mycobacterium tuberculosis $\mathrm{H}_{37} \mathrm{RV}$ was carried out by using standard procedure, microplate alamar blue assay (MABA) [46]. The MIC values of all the title compounds 7(a-o) along with standard drugs pyrazinamide and streptomycin for the comparison are summarized in Table-1. The MIC ranges in between 1.56 and $>100 \mu \mathrm{g} / \mathrm{mL}$.

Mycobacterium phlei: In the series of compounds 7(a-o), the chlorine or bromine on $6^{\text {th }}$ position of benzofuran exhibited equipotent activity (7k, 7m: MIC $1.56 \mu \mathrm{g} / \mathrm{mL})$, which were more potent than standard drugs pyrazinamide (MIC 3.125 $\mu \mathrm{g} / \mathrm{mL}$ ) and streptomycin (MIC $6.25 \mu \mathrm{g} / \mathrm{mL}$ ). Whereas, decrease

\begin{tabular}{|c|c|c|c|}
\hline \multicolumn{4}{|c|}{$\begin{array}{c}\text { TABLE-1 } \\
\text { RESULTS OF ANTITUBERCULAR ACTIVITY } \\
\text { OF COMPOUNDS 7(a-o) MICs }(\mu \mathrm{g} / \mathrm{mL})\end{array}$} \\
\hline Compound & $\mathrm{R}$ & $\begin{array}{c}\text { Mycobacterium } \\
\text { phlei }\end{array}$ & $\begin{array}{c}\text { Mycobacterium } \\
\text { tuberculosis } \\
\mathrm{H}_{37} \mathrm{RV} \\
\end{array}$ \\
\hline $7 a$ & $5-\mathrm{CH}_{3}$ & $>100$ & $>100$ \\
\hline $7 b$ & 6- $\mathrm{CH}_{3}$ & $>100$ & $>100$ \\
\hline $7 c$ & 4,6-di- $\mathrm{CH}_{3}$ & $>100$ & $>100$ \\
\hline 7d & $6,7-\mathrm{di}-\mathrm{CH}_{3}$ & $>100$ & $>100$ \\
\hline $7 e$ & 5-iso-Propyl & $>100$ & 50 \\
\hline $7 f$ & $5-t$-Butyl & $>100$ & 50 \\
\hline $7 \mathrm{~g}$ & $6-\mathrm{OH}$ & 12.5 & 12.5 \\
\hline $7 \mathrm{~h}$ & $5-\mathrm{OCH}_{3}$ & 12.5 & 25 \\
\hline $7 \mathbf{i}$ & $6-\mathrm{OCH}_{3}$ & 12.5 & 25 \\
\hline $7 \mathbf{j}$ & $5-\mathrm{Cl}$ & 3.125 & 6.25 \\
\hline $7 \mathbf{k}$ & $6-\mathrm{Cl}$ & 1.56 & 6.25 \\
\hline 71 & $5-\mathrm{Br}$ & 3.125 & 3.125 \\
\hline $7 \mathrm{~m}$ & $6-\mathrm{Br}$ & 1.56 & 3.125 \\
\hline $7 n$ & 4,5-Benzo & $>100$ & 25 \\
\hline 70 & 6,7-Benzo & $>100$ & 25 \\
\hline Pyrazinamide & - & 3.125 & 3.125 \\
\hline Streptomycin & - & 6.25 & 6.25 \\
\hline
\end{tabular}

in activity were observed by change in the position of chlorine or bromine from $6^{\text {th }}$ to $5^{\text {th }}$ position i.e., $7 \mathbf{j}, 7 \mathbf{l}$ : MIC $3.125 \mu \mathrm{g} / \mathrm{mL}$. Further, decrease in activity were observed on varying the substituent by hydroxy (7g), methoxy (7h, 7i) groups to MIC $12.5 \mu \mathrm{g} / \mathrm{mL}$. The least activity were observed for alkyl (7a-7f), benzo (7n, 7o) derivatives with MIC $>100 \mu \mathrm{g} / \mathrm{mL}$.

Mycobacterium tuberculosis $\mathbf{H}_{37} \mathbf{R V}$ : In the series of compounds $7(\mathbf{a}-\mathbf{0})$, the bromine either $5^{\text {th }}$ position or $6^{\text {th }}$ position on benzofuran exhibited equipotent activity (71, 7m: MIC 3.125 $\mu \mathrm{g} / \mathrm{mL}$ ), were equal to standard drug pyrazinamide (MIC 3.125 $\mu \mathrm{g} / \mathrm{mL}$ ) and more potent to streptomycin (MIC $6.25 \mu \mathrm{g} / \mathrm{mL}$ ). Whereas, decrease in activity were observed by changing the 
bromine by chlorine i.e., 7j, 7k: MIC $6.25 \mu \mathrm{g} / \mathrm{mL}$. The hydroxy derivative $7 \mathrm{~g}$ exhibited with MIC $12.5 \mu \mathrm{g} / \mathrm{mL}$, was more active than methoxy (7h, 7i) and benzo (7n, 7o ) derivatives with MIC $25 \mu \mathrm{g} / \mathrm{mL}$. Branched alkyl derivatives (7e, 7f) were exhibited MIC $50 \mu \mathrm{g} / \mathrm{mL}$, whereas mono-methyl $(\mathbf{7 a}, 7 \mathbf{b})$ and dimethyl (7c,7d) derivatives were least active in the series with MIC > $100 \mu \mathrm{g} / \mathrm{mL}$.

\section{Conclusion}

In conclusion, a series of novel benzofuran-oxadiazole conjugates $\mathbf{7}(\mathbf{a}-\mathbf{0})$ prepared were proved to be potential antitubercular agents against Mycobacterium phlei and Mycobacterium tuberculosis $\mathrm{H}_{37} \mathrm{RV}$. The compounds bearing chlorine $(\mathbf{7 j}, 7 \mathbf{k})$ and bromine $(\mathbf{7 l}, \mathbf{7 m})$ substituent of the benzofuran ring were found to be the most potent in the series. Among them, compound $7 \mathbf{m}$ bearing bromine on $6^{\text {th }}$ position on benzofuran was found to be most potent against both Mycobacterium phlei and Mycobacterium tuberculosis $\mathrm{H}_{37} \mathrm{RV}$. Further biological investigation is under progress in our laboratory and will be reported in due course.

\section{ACKNOWLEDGEMENTS}

The authors thank to the SAIF, Indian Institute of Technology Madras, Chennai, India for the ${ }^{1} \mathrm{H}$ NMR and ${ }^{13} \mathrm{C}$ NMR spectra; and USIC Karnatak University, Dharwad, India for IR, GCMS spectral data.

\section{CONFLICT OF INTEREST}

The authors declare that there is no conflict of interests regarding the publication of this article.

\section{REFERENCES}

1. WHO Global Tuberculosis Report (2018).

2. V. Bhowruth, L.G. Dover and G.S. Besra, Prog. Med. Chem., 45, 169 (2007);

https://doi.org/10.1016/S0079-6468(06)45504-1.

3. H. Lang, G. Quaglio and O. Olesen, Tuberculosis, 90, 1 (2010); https://doi.org/10.1016/j.tube.2009.10.002.

4. L.G. Dover and G.D. Coxon, J. Med. Chem., 54, 6157 (2011); https://doi.org/10.1021/jm200305q.

5. Beena and D.S. Rawat, Med. Res. Rev., 33, 693 (2013); https://doi.org/10.1002/med.21262.

6. K.M. Dawood, Expert Opin. Ther. Pat., 23, 1133 (2013); https://doi.org/10.1517/13543776.2013.801455.

7. R. Naik, D.S. Harmalkar, X. Xu, K. Jang and K. Lee, Eur. J. Med. Chem., 90, 379 (2015); https://doi.org/10.1016/j.ejmech.2014.11.047.

8. A. Radadiya and A. Shah, Eur. J. Med. Chem., 97, 356 (2015); https://doi.org/10.1016/j.ejmech.2015.01.021.

9. H. Khanam and Shamsuzzaman, Eur. J. Med. Chem., 97, 483 (2015); https://doi.org/10.1016/j.ejmech.2014.11.039.

10. R.J. Nevagi, S.N. Dighe and S.N. Dighe, Eur. J. Med. Chem., 97, 561 (2015);

https://doi.org/10.1016/j.ejmech.2014.10.085

11. A. Hiremathad, M.R. Patil, C. K. R, K. Chand, M.A. Santos and R.S. Keri, RSC Adv., 5, 96809 (2015); https://doi.org/10.1039/C5RA20658H.

12. V.N. Telvekar, A. Belubbi, V.K. Bairwa and K. Satardekar, Bioorg. Med. Chem. Lett., 22, 2343 (2012); https://doi.org/10.1016/j.bmcl.2012.01.067.

13. S.A. Galal, A.S. Abd El-All, M.M. Abdallah and H.I. El-Diwani, Bioorg. Med. Chem. Lett., 19, 2420 (2009); https://doi.org/10.1016/j.bmcl.2009.03.069.
14. L. Bigler, C. Spirli, R. Fiorotto, A. Pettenazzo, E. Duner, A. Baritussio, F. Follath and H.R. Ha, Eur. J. Med. Chem., 42, 861 (2007); https://doi.org/10.1016/j.ejmech.2006.12.031.

15. S. He, P. Jain, B. Lin, M. Ferrer, Z. Hu, N. Southall, X. Hu, W. Zheng, B. Neuenswander, C.H. Cho, Y. Chen, S.A. Worlikar, J. Aube, R.C. Larock, F.J. Schoenen, J.J. Marugan, T.J. Liang and K.J. Frankowski, ACS Comb. Sci., 17, 641 (2015);

https://doi.org/10.1021/acscombsci.5b00101.

16. Y. He, J. Xu, Z.H. Yu, A.M. Gunawan, L. Wu, L. Wang and Z.Y. Zhang, J. Med. Chem., 56, 832 (2013); https://doi.org/10.1021/jm301781p

17. A.M. Venkatesan, O. Dos Santos, J. Ellingboe, D.A. Evrard, B.L. Harrison, D.L. Smith, R. Scerni, G.A. Hornby, L.E. Schechter and T.H. Andree, Bioorg. Med. Chem. Lett., 20, 824 (2010); https://doi.org/10.1016/j.bmcl.2009.12.093.

18. H.F. Guo, H.Y. Shao, Z.Y. Yang, S.T. Xue, X. Li, Z.Y. Liu, X.B. He, J.D. Jiang, Y.Q. Zhang, S.Y. Si and Z.R. Li, J. Med. Chem., 53, 1819 (2010); https://doi.org/10.1021/jm901685n.

19. I.N. Gaisina, F. Gallier, A.V. Ougolkov, K.H. Kim, T. Kurome, S. Guo, D. Holzle, D.N. Luchini, S.Y. Blond, D.D. Billadeau and A.P. Kozikowski, J. Med. Chem., 52, 1853 (2009); https://doi.org/10.1021/jm801317h

20. S. Kumar, W. Namkung, A.S. Verkman and P.K. Sharma, Bioorg. Med. Chem., 20, 4237 (2012); https://doi.org/10.1016/j.bmc.2012.05.074.

21. S. Rizzo, C. Riviere, L. Piazzi, A. Bisi, S. Gobbi, M. Bartolini, V. Andrisano, F. Morroni, A. Tarozzi, J.P. Monti and A. Rampa, J. Med. Chem., 51, 2883 (2008); https://doi.org/10.1021/im8002747.

22. J. Suh, K.Y. Yi, Y.S. Lee, E. Kim, E.K. Yum and S. Yoo, Bioorg. Med. Chem. Lett., 20, 6362 (2010); https://doi.org/10.1016/j.bmcl.2010.09.102.

23. N. Negoro, S. Sasaki, S. Mikami, M. Ito, M. Suzuki, Y. Tsujihata, R. Ito, A. Harada, K. Takeuchi, N. Suzuki, J. Miyazaki, T. Santou, T. Odani, N. Kanzaki, M. Funami, T. Tanaka, A. Kogame, S. Matsunaga, T. Yasuma and Y. Momose, ACS Med. Chem. Lett., 1, 290 (2010); https://doi.org/10.1021/ml1000855.

24. J.C. Sacchettini, A. Aggarwal and M.K. Parai, Substituted Benzofuran Derivatives as Novel Antimycobacterial Agents, Patent WO 2016172498 A1 (2016).

25. K. Manna and Y.K. Agrawal, Eur. J. Med. Chem., 45, 3831 (2010); https://doi.org/10.1016/j.ejmech.2010.05.035.

26. J. Renuka, K.I. Reddy, K. Srihari, V.U. Jeankumar, M. Shravan, J.P. Sridevi, P. Yogeeswari, K.S. Babu and D. Sriram, Bioorg. Med. Chem., 22, 4924 (2014); https://doi.org/10.1016/j.bmc.2014.06.041.

27. B.R. Thorat, B. Nazirkar, V.B. Thorat, M. Mandewale, A. Nagarsekar and R.S. Yamgar J. Chem. Sci. Photon, 110, 279 (2016).

28. B. Nazirkar, U. Patil, B. Thorat, M. Mandewale, H. Gaokar, A. Pandhare and R. Yamgar, Der Pharm. Chemica, 9, 45 (2017).

29. S. Santoshkumar, N.D. Satyanarayana, R. Anantacharya and P. Sameer, Int. J. Pharm. Pharm. Sci., 9, 260 (2017); https://doi.org/10.22159/ijpps.2017v9i5.17564.

30. T. Aboul-Fadl, H.A. Abdel-Aziz, M.K. Abdel-Hamid, T. Elsaman, J. Thanassi and M.J. Pucci, Molecules, 16, 7864 (2011); https://doi.org/10.3390/molecules16097864.

31. B. Zhou, Y. He, X. Zhang, J. Xu, Y. Luo, Y. Wang, S.G. Franzblau, Z. Yang, R.J. Chan, Y. Liu, J. Zheng and Z.-Y. Zhang, Proc. Natl. Acad. Sci. USA, 107, 4573 (2010); https://doi.org/10.1073/pnas.0909133107.

32. L. Encinas, H. O'Keefe, M. Neu, M.J. Remuinan, A.M. Patel, A. Guardia, C.P. Davie, N. Perez-Macias, H. Yang, M.A. Convery, J.A. Messer, E. Perez-Herran, P.A. Centrella, D. Alvarez-Gomez, M.A. Clark, S. Huss, G.K. O’Donovan, F. Ortega-Muro, W. McDowell, P. Castaneda, C.C. Arico-Muendel, S. Pajk, J. Rullas, I. Angulo-Barturen, E. Alvarez-Ruiz, A. Mendoza-Losana, L.B. Pages, J. Castro-Pichel and G. Evindar, J. Med. Chem., 57, 1276 (2014); https://doi.org/10.1021/jm401326j.

33. S. Iqbal, M. Kesherwani, S. Dixit, D. Velmurugan and G. Krishnasamy, Int. J. Innov. Res. Computer Commun. Eng., 3, 11428 (2015).

34. P. Karunakar, C.R. Girija, V. Krishnamurthy, V. Krishna and K.V. Shivakumar, Tuberc. Res. Treat., 2014, Article ID 697532 (2014); https://doi.org/10.1155/2014/697532. 
35. B.R. Thorat, B. Nazirkar, V.B. Thorat, K. More, R. Jagtap and R. Yamgar, Asian J. Res. Chem, 9, 116 (2016); https://doi.org/10.5958/0974-4150.2016.00021.3.

36. B.R. Thorat, B. Nazirkar, V.B. Thorat, K. More, R. Jagtap and R. Yamgar, Asian J. Chem., 28, 2346 (2016);

https://doi.org/10.14233/ajchem.2016.19894.

37. M.J. Ahsan, J.G. Samy, C.B. Jain, K.R. Dutt, H. Khalilullah and M.S. Nomani, Bioorg. Med. Chem. Lett., 22, 969 (2012); https://doi.org/10.1016/j.bmcl.2011.12.014.

38. K.P. Harish, K.N. Mohana, L. Mallesha and B.N. Prasanna Kumar, Eur. J. Med. Chem., 65, 276 (2013); https://doi.org/10.1016/j.ejmech.2013.04.054.

39. D.R. Guda, S.J. Park, M.W. Lee, T.J. Kim and M.E. Lee, Eur. J. Med. Chem., 62, 84 (2013); https://doi.org/10.1016/j.ejmech.2012.12.035.

40. H. Rajak, B. Singh Thakur, A. Singh, K. Raghuvanshi, A.K. Sah, R. Veerasamy, P.C. Sharma, R. Singh Pawar and M.D. Kharya, Bioorg. Med. Chem. Lett., 23, 864 (2013); https://doi.org/10.1016/j.bmcl.2012.11.051.

41. N.C. Desai, N. Bhatt, H. Somani and A. Trivedi, Eur. J. Med. Chem., 67, 54 (2013);

https://doi.org/10.1016/j.ejmech.2013.06.029.
42. J. Sun, H. Zhu, Z.M. Yang and H.L. Zhu, Eur. J. Med. Chem., 60, 23 (2013); https://doi.org/10.1016/j.ejmech.2012.11.039.

43. M. Neelgundmath, K.R. Dinesh, C.D. Mohan, F. Li, X. Dai, K.S. Siveen, S. Paricharak, D.J. Mason, J.E. Fuchs, G. Sethi, A. Bender, K.S. Rangappa, O. Kotresh and Basappa, Bioorg. Med. Chem. Lett., 25, 893 (2015); https://doi.org/10.1016/j.bmcl.2014.12.065.

44. S.M. Hiremath, A. Suvitha, N.R. Patil, S.S. Khemalapure, C.S. Hiremath, S.K. Pattanayak, V.S. Negalurmath, K. Obelannavar, S.J. Armakovic and S. Armakovic, Spectrochim. Acta A Mol. Biomol. Spectrosc., 205, 95 (2018); https://doi.org/10.1016/j.saa.2018.07.003.

45. V.S. Negalurmath, S.K. Boda, O. Kotresh, P.V. Anantha Lakshmi and M. Basanagouda, Chemical Data Collections, 19, 100178 (2019); https://doi.org/10.1016/j.cdc.2019.100178.

46. M.C.S. Lourenco, M.V.N. de Souza, A.C. Pinheiro, M.L. Ferreira, R.S.B. Goncalves, T.C.M. Nogueira and M.A. Peralta, ARKIVOC, 15, 181 (2007); https://doi.org/10.3998/ark.5550190.0008.f18. 